\title{
Improvement in Data Acquisition for a Step-Scan Fourier Transform Spectrometer
}

\author{
THOU-LONG CHIN and KING-CHUEN LIN * \\ Department of Chemistry, National Taiwan University, and Institute of Atomic and Molecular Sciences, Academia Sinica, Taipei \\ 106, Taiwan, Republic of China
}

\begin{abstract}
We have designed a controller circuit to be incorporated into a commercial step-scan Fourier transform spectrometer (Bruker IFS 88 FTS). Alternatively, time-resolved data may be acquired with a transient digitizer provided by the user. We have demonstrated the versatility of the controller with a Lecroy $9450 \mathrm{~A}$ transient digitizer as the recorder in the time-resolved emission detection of the $\mathrm{CH}$ radical populated in the $A^{2} \Delta$ state and in the absorption measurement of the ambient air. The controller, which may be conveniently coupled to any data acquisition system, makes the use of the FTS more flexible and less expensive.
\end{abstract}

Index Headings: Step-scan Fourier transform spectrometer; Interferogram; Data acquisition; $\mathrm{CH}$ emission spectrum.

\section{INTRODUCTION}

Since the first step-scan Fourier transform spectrometer (FTS) from Bruker (IFS 88 Model) was commercialized in 1987, the instrument has been increasingly used to study dynamic phenomena. ${ }^{1-3}$ With the advent of the step-scan FTS, it becomes possible for time-resolved FT-infrared (FT-IR) to achieve time resolution ranging from nanoseconds to milliseconds. In contrast to the conventional rapid-scan method, the moving mirror in the step-scan FTS is moved step by step rather than in a continuous fashion. Feedback from the reference $\mathrm{HeNe}$ laser interference pattern is used to control the mirror position rather than the mirror velocity. In the operation of the step-scan technique, while the mirror is moved to a fixed position, a repeatable phenomenum is initiated and the time evolution of the event is recorded at that position. The procedure is repeated at the next mirror position. After the moving mirror has completed a whole run of retardation positions, the collected interferograms corresponding to different time slices are Fourier transformed to yield time-resolved spectra. ${ }^{1-3}$ The subsequent time resolution essentially depends only on the signal strength, the detection sensitivity, and the response speed of the detector and the data acquisition electronics.

Thus far, the step-scan FTS has been widely applied in different research such as investigations of the reorientation of polymers and liquid crystals, associated with mechanical or electric field perturbation, ${ }^{1,4-6}$ and dynamic behavior of a ligand binding to a transition metal element or a heme. ${ }^{1}$ Gerwert and co-workers employed step-scan FT-IR difference spectroscopy to study membrane protein bacteriorhodopsin and resolved for the first time the biphasic rise of bacteriorhodopsin's intermediate in the infrared spectral region. ${ }^{7}$ Yang investigated the effectiveness of various metal catalysts in anaerobic composition

Received 16 June 1998; accepted 21 September 1998.

* Author to whom correspondence should be sent. as a function of time. ${ }^{8}$ Photoacoustic (PA) signal measurement performed with an FT-IR spectrometer has been widely accepted. In the conventional rapid-scan mode, however, a problem arises in the interpretation of the depth information from PA measurements. ${ }^{1,9}$ With stepscan FT-IR, Palmer and co-workers demonstrated the capability of mapping depth profiles from different layers in terms of different phase characteristics between these substances. ${ }^{1,10}$ Later, Budsvska and Manning found that time-resolved impulse photoacoustic spectra may straightforwardly provide depth-profiling information for solid samples. ${ }^{9}$ The step-scan technique has also been extended to the study of gas-phase kinetics and dynamics. Time-resolved FT emissions or absorptions in the infrared or visible range have been measured in order to look into the photofragmentation process, single collision reactions, energy transfer events, and dynamics and kinetics of radical-radical reactions initiated by a photolysis laser. ${ }^{2,3,11-16}$ The research activities involving the step-scan FTS continue to increase.

The data acquisition system in the step-scan FTS from Bruker (IFS 88 Model) depends mainly on two transient recorder boards. One is a $200 \mathrm{kHz}, 16$ bit analog-to-digital converter (ADC) and the other is a $200 \mathrm{MHz}, 8$ bit PAD 82 board. ${ }^{17}$ For events in the microsecond time domain, the tiny signal may be effectively acquired by using the 16 bit ADC recorder. However, as the phenomena are short in the nanosecond region, the provision of the $8 \mathrm{bit}$, $1 \mathrm{~V}$ range PAD 82 recorder is sometimes insufficient to record a trace signal without distortion. When the strength of the analog signal is less than 1/256 voltage, the response is essentially lost in the analog-to-digital conversion. Such a small signal may often be found in retardation positions away from the centerburst of an interferogram. As a result, the spectral resolution is sacrificed, since the interference pattern beyond that position contributes insignificantly. Another disadvantage for the current Bruker IFS 88 is that the generated interferogram in the step-scan mode cannot be retrieved again. The FTS operated in the step-scan mode may generate much more data than in the rapid-scan mode, since an additional time coordinate is included. It becomes a heavy burden to save all the interferograms. Nevertheless, these data may be manipulated to produce different outcomes. They are also useful in examining the quality of the spectra prior to Fourier transformation.

This work is intended to provide a controller circuit that will enable users to select their own data acquisition system as an alternative to the present commercial one. This circuitry can retrieve the interference signal in each mirror position in a way that is synchronous with the 


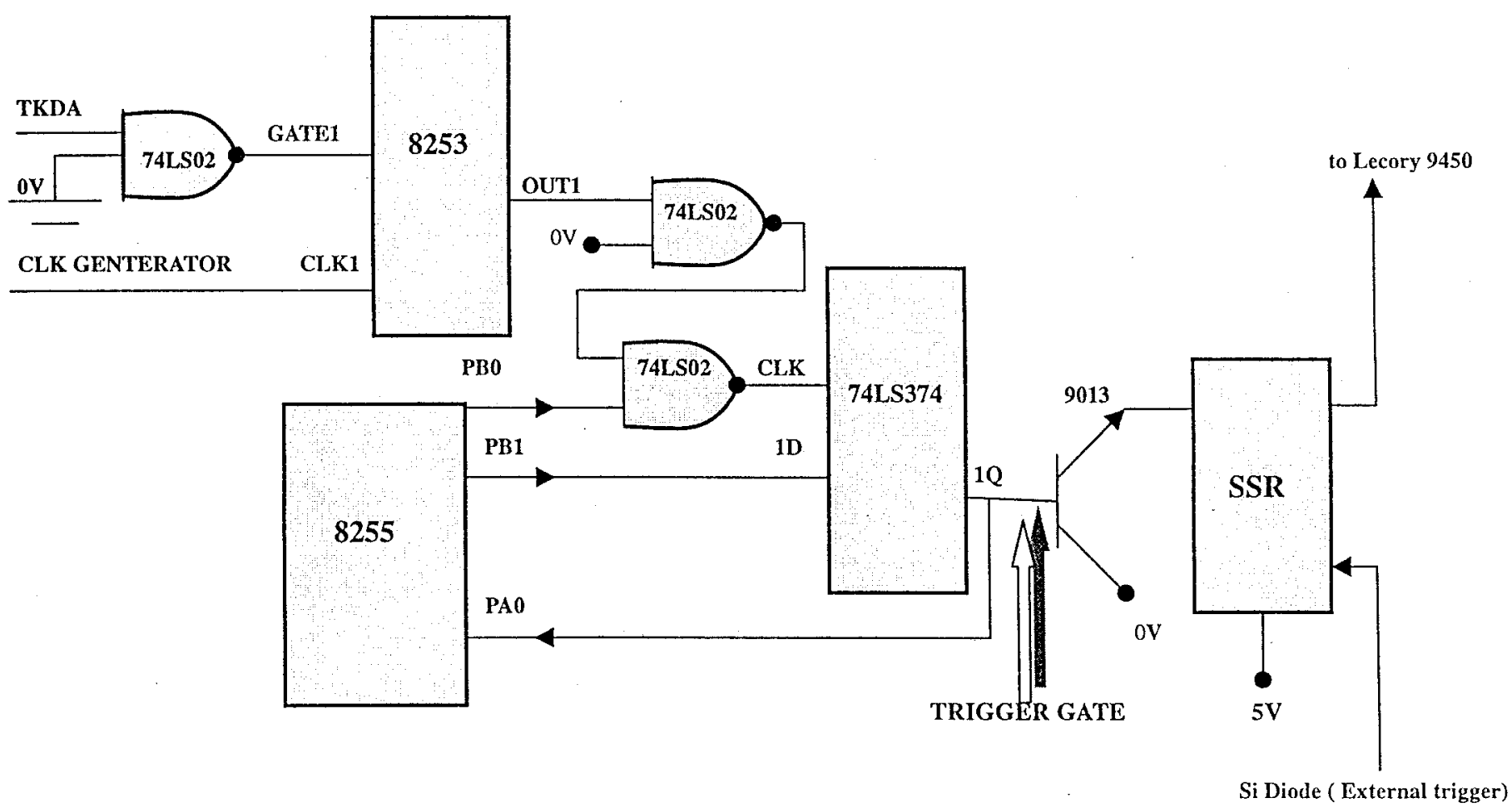

FIG. 1. Schematic diagram of controller circuit. The notations are described in the text.

commercial time-resolved spectrometer (TRS). The data are acquired alternatively with a Lecroy $9450 \mathrm{~A}$ transient digitizer or any other available recorder. The signal processing can be made more flexible. The Fourier transformed spectra are even better in terms of the spectral resolution and the signal-to-noise ratio than is the case with the current TRS provided in the IFS 88 apparatus. With the above controller and recorder, two experiments are demonstrated. One is the time-resolved FT emission spectrum of the $\mathrm{CH}$ radical populated in the $\mathrm{A}\left({ }^{2} \Delta\right)$ state, and the other is the absorption spectrum of ambient air.

\section{EXPERIMENT AL}

Design of Controller. In the operation of the stepscan mode of the IFS 88 instrument, when the moving mirror is in the process of moving, a TKDA pin (on a CDP board) sends a negative pulse with a 20 ms duration, causing the system to stop taking data. ${ }^{17}$ Thus the action triggering the transient recorder for data acquisition is ignored. In contrast, when the mirror has been moved to a fixed position and stabilized, the TKDA sends out a 0.1 ms pulse, notifying the system to start taking data. Therefore, monitoring the TKDA signals is an effective way to determine the state of the step-scan FTS. The controller is designed on the basis of this concept.

As illustrated in Fig. 1, the circuitry is assembled with 8253 (programable timer/counter) and 8255 (programable peripheral interface) chips, which are mounted on the PC I/O board, NOR gate (74LS02), D-type flip-flop (74LS374), transistor (9013), and solid-state relay (SSR). The total cost of the controller is less than $\$ 100$. The operation procedure follows. The TKDA negative pulse is first received from the IFS 88 instrument and then inverted to a positive pulse with a NOR gate (74LS02).
The pulse and a clock generated from a function generator are input to an 8253 chip, which is set to mode 5, the so-called hardware triggered strobe. ${ }^{18}$ The function of the 8253 chip is to recognize the status of the moving mirror. When the $20 \mathrm{~ms}$ TKDA pulse is met, a clock inside the 8253 chip begins to count down from $n$ to 0 , then gives a pulse (or clock) within the $20 \mathrm{~ms}$ period. The $n$ value should be large enough so that the counting action will not finish during the duration of the $0.1 \mathrm{~ms}$ TKDA pulse. In this manner, the 8253 chip may ignore the signal of the $0.1 \mathrm{~ms}$ pulse but respond with a pulse (or clock) when the $20 \mathrm{~ms}$ pulse is encountered. The pulse sent from the 8253 chip can be inverted through the NOR gate, since outport pB0 of the 8255 chip is at $\operatorname{logic} 0$. The 8255 chip is set at mode 0 . That is, the ports $\mathrm{pA}, \mathrm{pB}$, and $\mathrm{pC}$ may be used as inport and outport independently. ${ }^{18}$ If the $\mathrm{pB} 1$ is at logic 1 , then the outport Q1 of the D-type flip-flop (74LS374), while activated by the input clock, responds with logic 1 . The output voltage is then amplified by a transistor (9013) to an extent that is sufficient to turn off the SSR. Accordingly, the output of a Si diode fails to simultaneously activate the transient digitizer (Lecroy 9450A) provided externally and the TRS recorders in the IFS 88 . The $\mathrm{Si}$ diode is used to monitor the laser pulse (or other external perturbers), serving as a triggering source for the transient digitizers to take data. A polling line connected to the outport Q1 is fed back to the 8255 chip. Once the polling line receives the signal of logic 1 , a command is sent to the host PC (provided by the users) to start to read and save the previous data left on the Lecroy $9450 \mathrm{~A}$. The data transfer is executed through an IEEE 488 interface. Right after completion of the data transfer, the outport $\mathrm{pB} 1$ changes the logic from 1 to 0 , and then outport $\mathrm{pB} 0$ gen- 


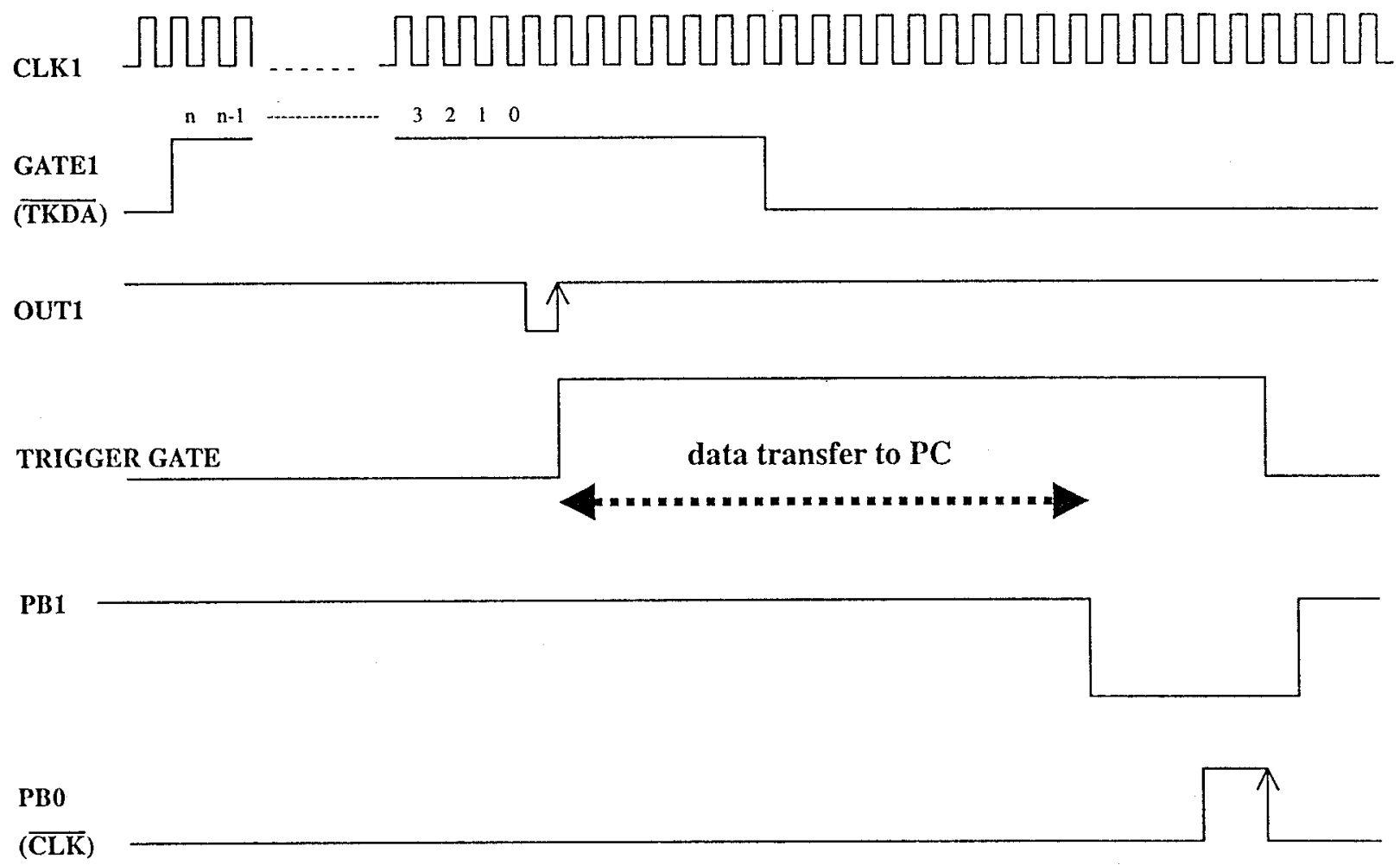

FIG. 2. The time sequence of the controller operation.

erates a clock. As the flip-flop (74LS374) is activated again by the positive transition of the clock, the Q1 changes the output from logic 1 to 0 . At that moment, the signal of logic 0 may turn on the SSR, and then a pulse from the Si diode simultaneously triggers the Lec- roy $9450 \mathrm{~A}$ and TRS recorder of the FTS. These recorders start to take data from the new position of a moving mirror as soon as it is stabilized. The data recording may be averaged over a number of laser shots. While the mirror is moving to the next step, the TKDA emits a $20 \mathrm{~ms}$

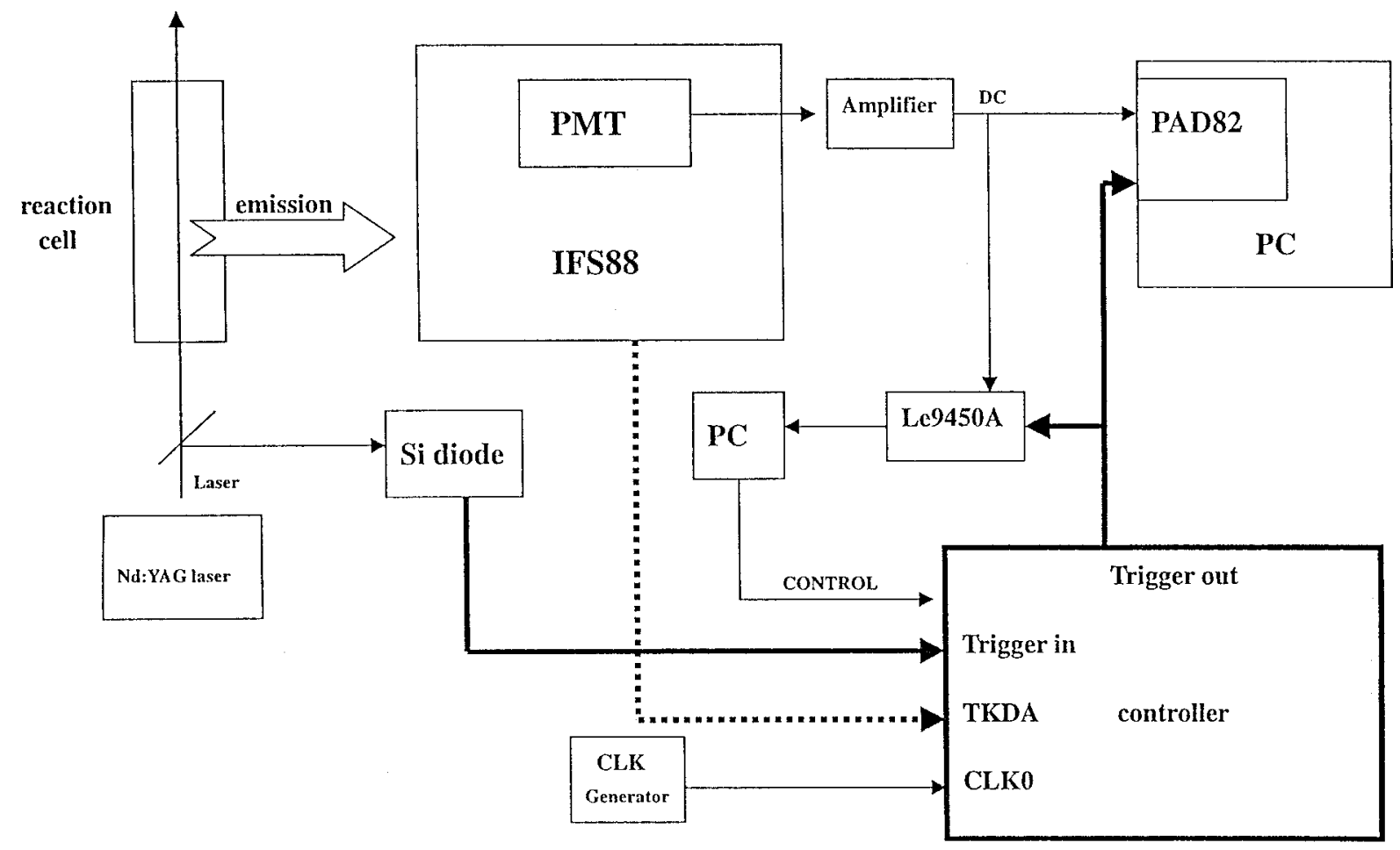

FIG. 3. Experimental setup for time-resolved FT emission of the $\mathrm{CH}$ radical populated in the $\mathrm{A}\left({ }^{2} \Delta\right)$ state. The data may be acquired by PAD 82 and Lecroy $9450 \mathrm{~A}$ recorders simultaneously through the controller device interfaced to the IFS 88 instrument. 


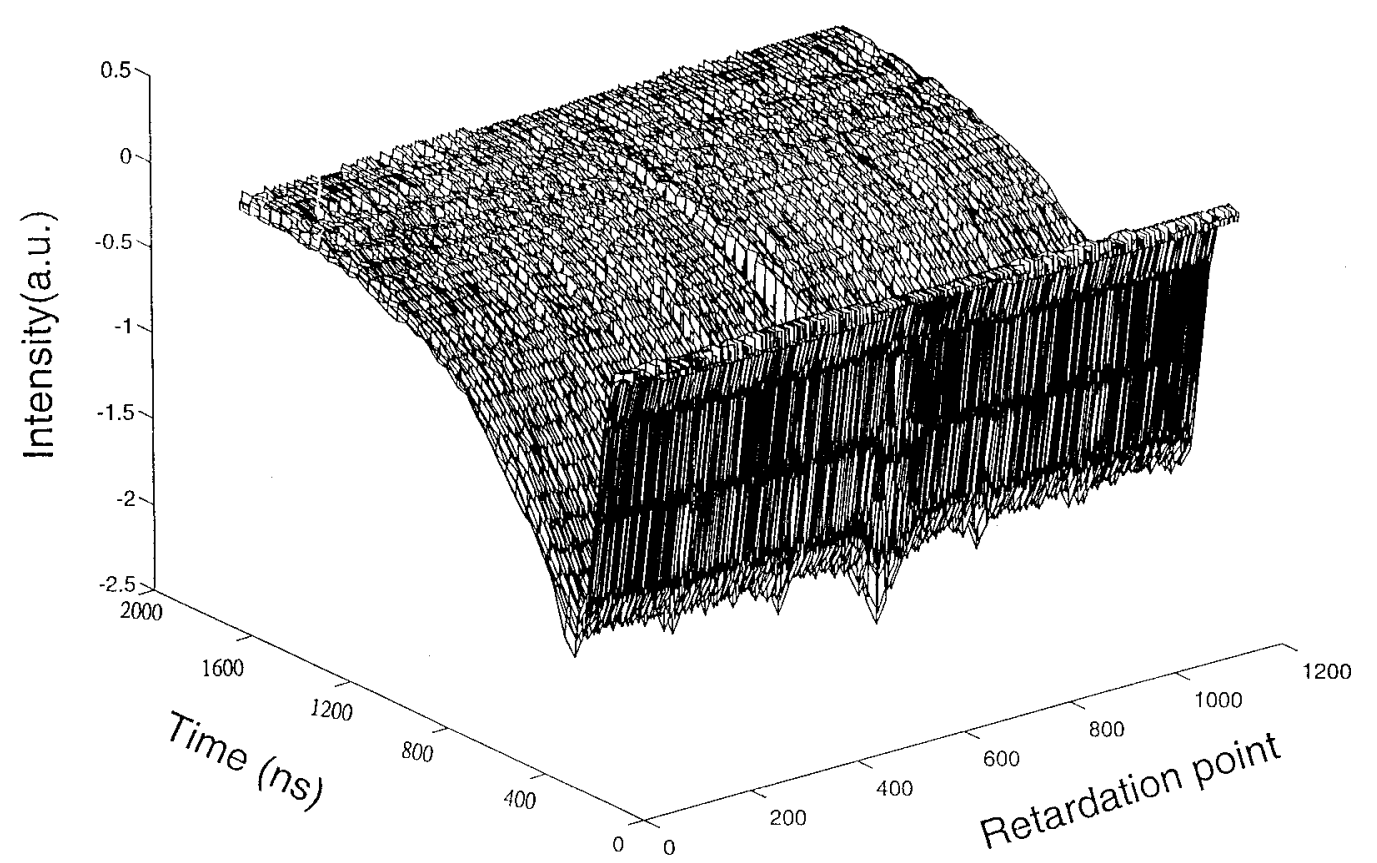

FIG. 4. The interference waveforms from $\mathrm{CH} \mathrm{A}\left({ }^{2} \Delta\right)$ emission as a function of retardation position recorded with the Lecroy $9450 \mathrm{~A}$ transient digitizer. The waveform ranges in the time domain from 0 to $1780 \mathrm{~ns}$ with a temporal resolution of $2.5 \mathrm{~ns}$.

pulse again. The above procedures then repeat. For clarity, the resultant time sequence for the controller operation is depicted in Fig. 2.

Experimental Procedure. Time-Resolved FT Emission. The controller device has been successfully applied to the following two experiments. One is for the timeresolved FT emission study of the $\mathrm{CH} \mathrm{A}\left({ }^{2} \Delta\right)$ state, from which the rates of rotational and vibrational energy transfer may be evaluated. ${ }^{16}$ The experimental apparatus is shown in Fig. 3. The $\mathrm{CH}$ radical in the $\mathrm{A}\left({ }^{2} \Delta\right)$ state was produced following photolysis of $\mathrm{CHBr}_{3}$ at $266 \mathrm{~nm}$. The dissociating laser pulse came from a $10 \mathrm{~Hz}, 5-8 \mathrm{~ns} \mathrm{Nd}$ : YAG laser (Spectra-Physics, GCR3) operating in the fourth harmonic. The precursor flowed through the reaction cell, so that emission from the fresh $\mathrm{CH}$ radical was monitored. The emission spectrum from 420.2 to $434.8 \mathrm{~nm}$ of the $\mathrm{CH} \mathrm{A}\left({ }^{2} \Delta\right)$ state was collected through a pair of lenses of 2 in. $(f / 1.5)$ and 6 in. $(f / 4)$ focal lengths onto the entrance of a step-scan Fourier transform spectrometer. A fraction of the laser beam was sent to irradiate a rodamine $6 \mathrm{G}$ dye contained in a cuvette. The resulting fluorescence was monitored with a $\mathrm{Si}$ diode, which was connected to the external controller, as described above. The emission of the $\mathrm{CH} A\left({ }^{2} \Delta\right)$ state through a Michelson interferometer was detected by a photomultiplier tube (RCA, 1P28A). The dc-coupled signal was amplified before being fed into the PAD 82 and Lecroy $9450 \mathrm{~A}$ transient digitizers simultaneously. The resulting data from either the PAD 82 or Lecroy $9450 \mathrm{~A}$ were treated with a separate PC.

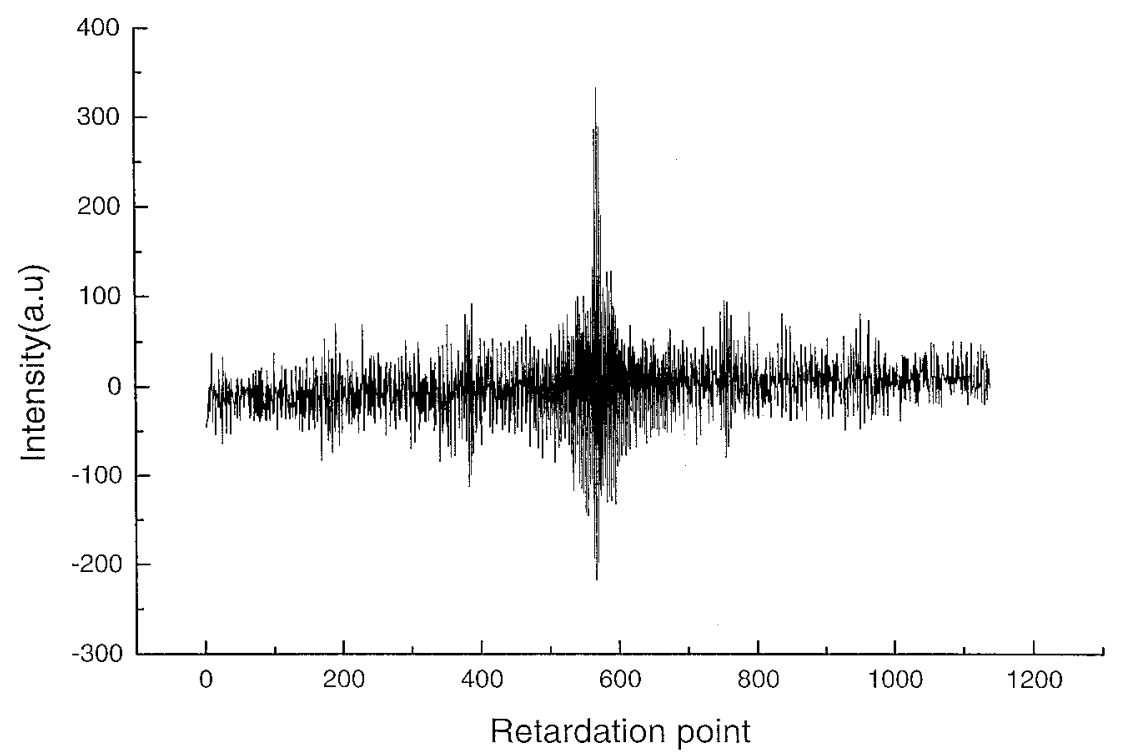

FIG. 5. The interferogram corresponding to a particular time slice given in Fig. 4. 


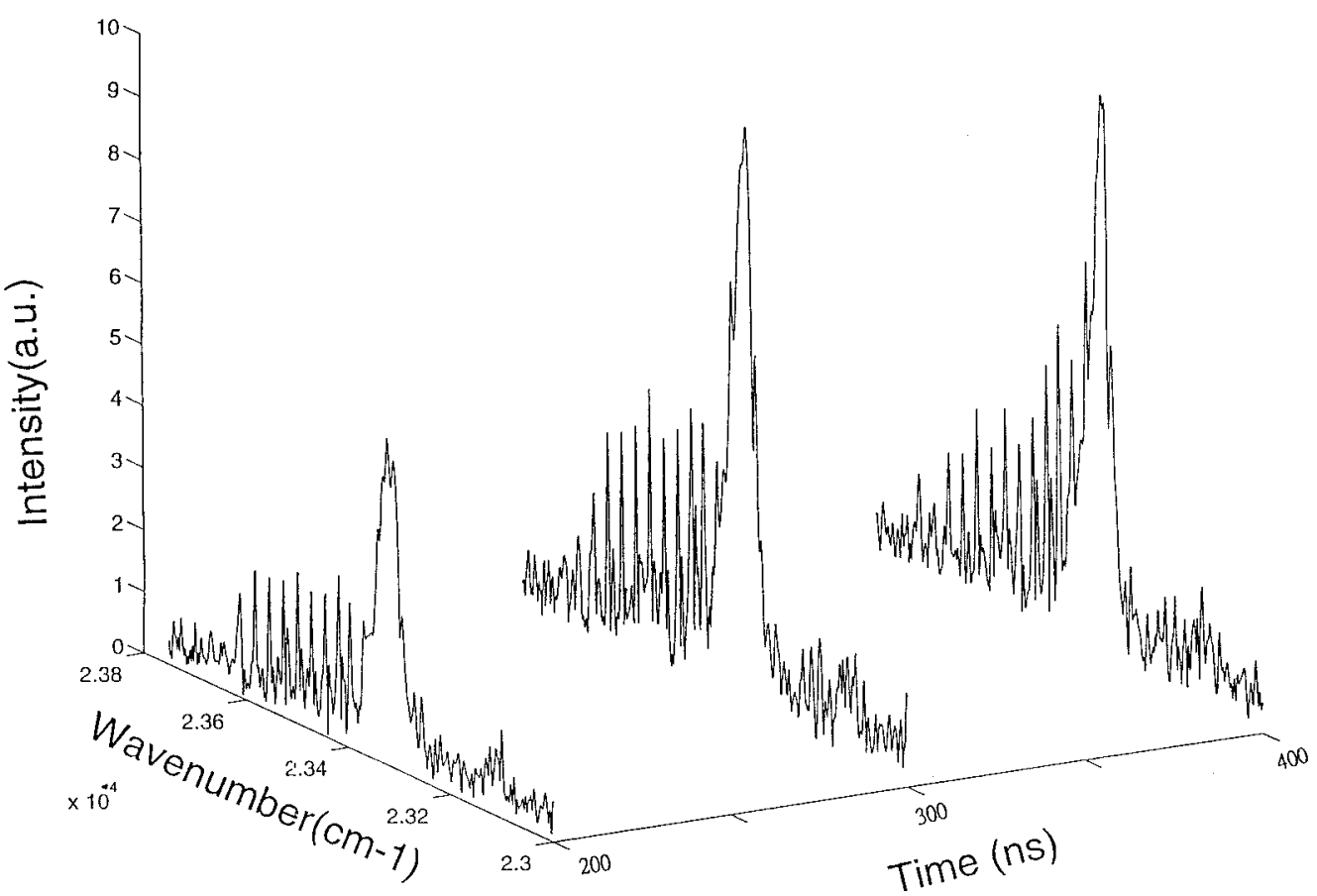

FIG. 6. The time-resolved FT emission spectra of the $\mathrm{CH} \mathrm{A}\left({ }^{2} \Delta\right)$ state corresponding to three time slices given in Fig. 4.

Time-Resolved FT Absorption. The controller was also applied to the time-resolved FT-IR absorption experiment on the ambient air. A standard setup for step-scan FT-IR was employed. A globar source irradiated through the Michelson interferometer, and the resulting interference pattern after passing through the ambient air in the chamber was detected with a fast-response MCT (Model No. KMPV11-1-J2, Kolmar Technologies). The triggering source of the Si diode was replaced with a function generator. The procedure for the controller operation was the same as in the emission experiment.

\section{RESULTS AND DISCUSSION}

CH(A) Emission. As shown in Fig. 4, the interference waveforms from the $\mathrm{CH} \mathrm{A}\left({ }^{2} \Delta\right)$ emission were recorded in different retardation positions with the use of the Lec-

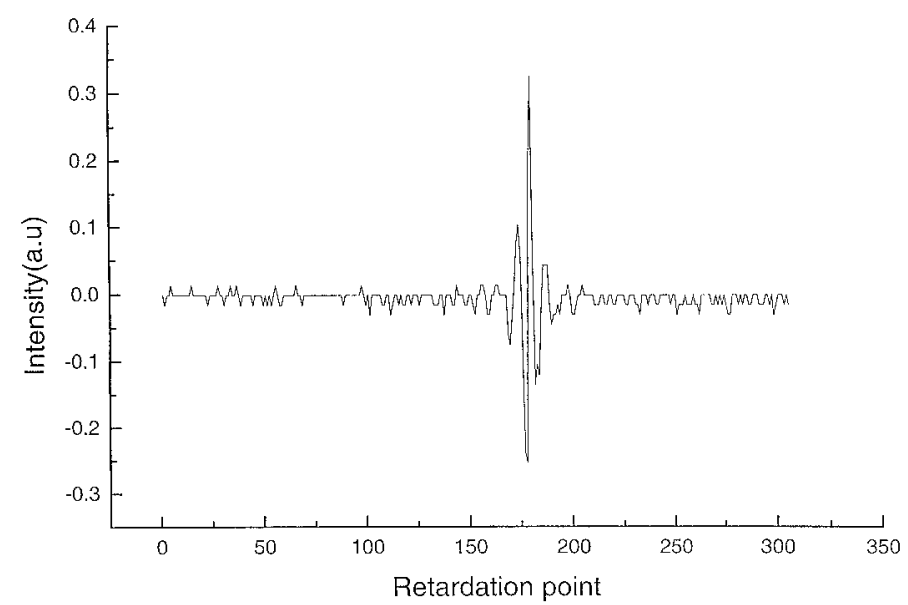

FIG. 7. The interferogram for ambient air absorption acquired in stepscan mode with the use of the Lecroy $9450 \mathrm{~A}$ recorder with the voltage gain fixed. roy 9450 A. Each profile was averaged over 10 laser shots to improve the signal-to-noise ratio. An example of the interferogram corresponding to a particular time slice (120 ns time delay) is given in Fig. 5. Some of the resulting spectra after Fourier transformation are shown in Fig. 6. The spectral and time resolution were adjusted to be $10 \mathrm{~cm}^{-1}$ and $2.5 \mathrm{~ns}$, respectively.

The Lecroy $9450 \mathrm{~A}$ employed in this work has a better voltage resolution than the PAD 82, although both recorders have the same resolution of 8 bits. The Lecroy $9450 \mathrm{~A}$ has a selectable range of 0 to $20 \mathrm{~V}$ and selectable gain of $1,2,4,10,20,40,100,200,400$, or 5000 . With a voltage of 0 to $20 \mathrm{~V}$ and a gain of 5000 , the smallest detectable change in voltage is $1.6 \times 10^{-5} \mathrm{~V}$, which means that the Lecroy $9450 \mathrm{~A}$ is theoretically capable of recording the signals down to $1.6 \times 10^{-5} \mathrm{~V}$. In contrast,

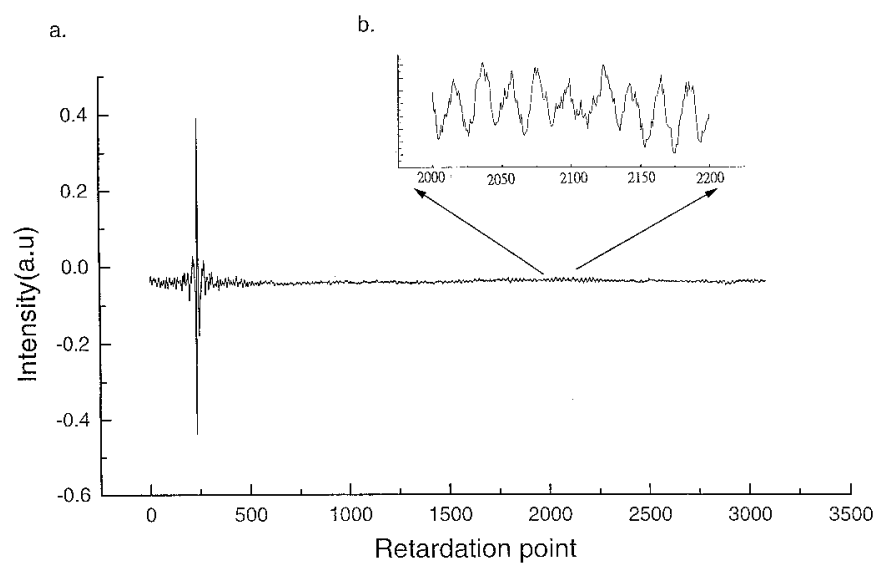

FIG. 8. (a) The interferogram for ambient air absorption acquired in step-scan mode with the use of the Lecroy 9450A recorder with the voltage gain adjusted appropriately. (b) The enlarged region for the retardation points from 2000 to 2200 . 


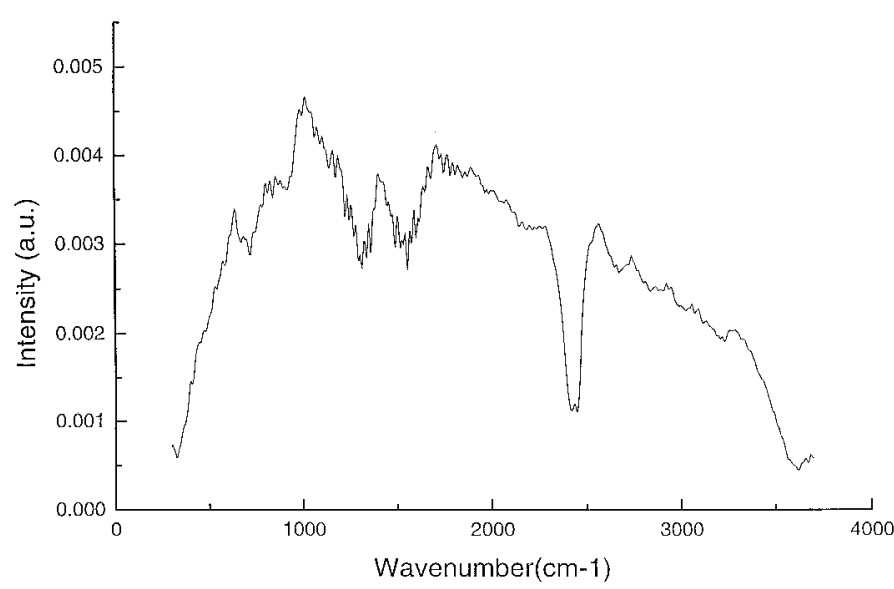

FIG. 9. Fourier transformed spectrum of ambient air corresponding to the interferogram in Fig. 8.

the PAD 82 recorder has a voltage range of 0 to $1 \mathrm{~V}$ and a fixed gain of 1 ; the smallest detectable change is therefore $3.9 \times 10^{-3} \mathrm{~V}$. Thus, the theoretical resolution of one bit for Lecroy $9450 \mathrm{~A}$ is about two orders of magnitude better than that for the PAD 82 recorder. Accordingly, the time-resolved FT spectra recorded by the Lecroy $9450 \mathrm{~A}$ are expected to have better quality than results from the PAD 82 recorder.

The current IFS 88 model does not save the data of interferograms in the step-scan mode. Once the interferograms are Fourier transformed, these data are deleted from the memory. Thus one cannot retrieve the corresponding interferogram to inspect the data quality or to further manipulate the data. In addition, if the spectral resolution is increased, one has to reduce the full range of spectral wavelength to keep the memory space unsaturated. In contrast, with our external controller incorporating the Lecroy $9450 \mathrm{~A}$, the acquired interference waveform at a particular retardation position can be successively removed to the PC during the period when the mirror is moving to the next position. Therefore, the space restriction may be solved with provision of a large RAM and hard disk. Let us estimate the memory space for taking the data given in Figs. 4-6. The Lecroy 9450A provides a temporal resolution of $2.5 \mathrm{~ns}$. The profile (Fig. 4) spreads in the time domain from 0 to $1710 \mathrm{~ns}$, containing 684 sampling points. The resulting data may occupy a memory space of 2 kbyte in the Lecroy 9450A. Each profile, averaged over 10 laser shots, takes only 5 $\mathrm{ms}$ to transfer to the host $\mathrm{PC}$ with a transmission rate of

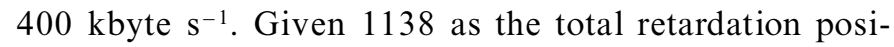
tions, corresponding to a spectral resolution $10 \mathrm{~cm}^{-1}$, the storage space needs to be about 2 Mbyte. Such space may easily be found in our PC. In comparison, we also estimate the memory space for the IFS 88 to accommodate the interference waveforms in Fig. 4. According to the formula given in the OPUS manual, the evaluated memory space corresponds to 7.8 Mbyte. ${ }^{17}$ This value is far beyond the space allowance of 4 Mbyte RAM provided. On the other hand, because of no restrictions in the memory space, a single Lecroy $9450 \mathrm{~A}$ is able to measure the whole dynamic phenomena ranging from nanoseconds to milliseconds. Of course, that treatment is at the expense of more time spent in manipulating the data. To prune the huge amount of data accumulated in the study of the reaction mechanism of membrane protein bacteriorhodopsin, Gerwert and co-workers simultaneously employed two transient recorders in a step-scan FT-IR difference absorbance experiment. ${ }^{7}$ One 8 bit, $200 \mathrm{MHz}$ recorder was used to measure the time domain from 100 ns to $20 \mu \mathrm{s}$, while the other $12 \mathrm{bit}, 200 \mathrm{kHz}$ recorder monitored the $5 \mu$ s to $\sim 50 \mathrm{~ms}$ time range.

Ambient Air Absorption. The ambient air absorption experiment in the step-scan mode is demonstrated to examine the capability of measuring a broad band using an 8 bit transient recorder. The interferogram for a broadband spectrum usually causes a sharp contrast in signal strength between the centerburst and the position away from it. The use of an 8 bit transient recorder may sometimes cause the trace signal acquired away from the centerburst to be ignored. An example is shown in Fig. 7. The interferogram for the ambient air was measured with the Lecroy $9450 \mathrm{~A}$ with the voltage gain purposely fixed. The weak signal in the figure was seriously distorted. In comparison, Fig. 8 shows the corresponding interferogram from the same recorder with the voltage gain adjusted appropriately. The weak signal was enlarged by changing the gain. Therefore, the interference pattern even at retardation points that are far away may be recognizable. The resulting Fourier transformed spectrum (Fig. 9) may maintain the spectral resolution as expected in the initial condition. In contrast to the Lecroy $9450 \mathrm{~A}$ measurement, the 8 bit, $200 \mathrm{MHz}$ PAD 82 recorder lacks flexibility in the adjustment of the voltage gain. The resulting difficulty in recognizing the whole interference pattern may reduce the spectral resolution.

\section{CONCLUSION}

In conclusion, we have designed a circuit controller to be incorporated into a step-scan FTS (IFS 88). The time-resolved data may be acquired alternatively with a transient digitizer provided by the user. By taking advantage of the Lecroy $9450 \mathrm{~A}$ as a recorder, we have demonstrated the versatility of the controller in measuring time-resolved FT emission and absorption spectra. The controller, which may feasibly be coupled to any data acquisition system, makes the use of the FTS more flexible and less expensive.

\section{ACKNOWLEDG MENTS}

The authors wish to thank J. F. Nien, D. K. Liu, and Y. P. Chen for help in setting up the emission experiment. This work is supported by the National Science Council and Petroleum Company of the Republic of China under Contract No. NSC87-2119-M-002-001.

1. R. A. Palmer, J. L. Chao, R. M. Dittmar, V. G. Gregoriou, and S. E. Plunkett, Appl. Spectrosc. 47, 1297 (1993).

2. G. V. Hartland, W. Xie, and H. L. Dai, Rev. Sci. Instrum. 63, 3261 (1992).

3. T. J. Johnson, A. Simon, J. M. Weil, and G. W. Harris, Appl. Spectrosc. 47, 1376 (1993).

4. K. Masutani, H. Sugisawa, A. Yokota, Y. Furukawa, and M. Tasumi, Appl. Spectrosc. 46, 560 (1992).

5. M. A. Czarnecki, B. Jordanov, S. Okretic, and H. W. Siesler, Appl. Spectrosc. 51, 1698 (1997).

6. H. Wang, R. A. Palmer, and C. J. Manning, Appl. Spectrosc. 51, 1245 (1997).

7. R. Rammelsberg, B. Hebling, H. Chorongiewski, and K. Gerwert, Appl. Spectrosc. 51, 558 (1997). 
8. D. B. Yang, Appl. Spectrosc. 47, 1425 (1993).

9. B. O. Budevska and C. J. Manning, Appl. Spectrosc. 50, 939 (1996).

10. R. M. Dittmar, J. L. Chao, and R. A. Palmer, Appl. Spectrosc. 45, 1104 (1991).

11. S. A. Rogers and S. R. Leone, Appl. Spectrosc. 47, 1430 (1993).

12. S. R. Leone, Acc. Chem. Res. 22, 139 (1989).

13. D. E. Heard, R. A. Brownsword, D. G. Weston, and G. Hancock, Appl. Spectrosc. 47, 1438 (1993).
14. G. Hancock and D. E. Heard, in Advances in Photochemistry (John Wiley and Sons, New York, 1993), Vol. 18, pp. 1-65.

15. J. Eberhard, P. S. Yeh, and Y. P. Lee, J. Chem. Phys. 107, 6499 (1997).

16. C. C. Wang, T. L. Chin, and K. C. Lin, J. Chem. Phys. 107, 10348 (1997).

17. "Step-Scan Technical Manual for IFS 66, IFS 66v and IFS 88" (Bruker, Karl Sruhe, Germany).

18. Intel Microsystem Components Handbook Vol. II (Intel, Folsom, California). 